\title{
Morinda Citrifolia (Noni) in the Treatment of Psoriasis
}

\author{
Hiroshi Okamoto*
}

Minami-otsuka Institute of Technology, Minami-otsuka Clinic, Tokyo, Japan

\begin{abstract}
Morinda citrifolia (Noni) fruits have been used traditionally to treat diabetes, high blood pressure, cancer, mechanical injury, and arthritis. However its mechanism of action have not yet been fully elucidated. Herein I describe an important case in which psoriasis was treated with $M$. citrifolia in combination with conventional anti-rheumatism therapy. This is the first report showing the effect of M. citrifolia on skin lesions associated with psoriasis. This case sheds light on the pathological difference between skin lesions and arthropathy associated with psoriasis.
\end{abstract}

Keywords: M. citrifolia, anti-rheumatism therapy, interleukin(IL)-4, MTX, DAS28-CRP.

\section{Text}

Morinda citrifolia (Noni) is a small to medium-sized tree that grows in India, Burma, China, and Polynesian islands. $M$. citrifolia fruits have been used traditionally to treat diabetes, high blood pressure, cancer, mechanical injury, and arthritis by native Polynesians. However, in addition to the lack of clinical evidence, the mode of actions of this fruit have not yet been elucidated..Several researchers have reported that the immune-stimulant properties of $M$. citrifolia contribute to its anti-tumor potential [1]. M. citrifolia juice concentrate has been reported to stimulate the cannabinoid receptors, decrease interleukin(IL)-4 levels and increase interferon- $\gamma$ levels pointing to the immunostimulant potentialof this plant. In addition, a polysaccharide-rich substance was reported to stimulate the release of tumor necrosis factor- $\alpha$, IL-1 $\beta$, IL-10, IL-12 p70 and nitric oxide.Recently, the hydroalcoholic extract and one of the fractions of $M$. citrifolia fruits significantly enhanced the antibody titer in immunecompromised animals [2]. In the study with human fibroblasts, anthraquinone isolated from $M$. citrifolia showed significantly increased elaboration of procollagen type I C terminal peptide and glycosaminoglycans, as well as reduced expression of the collagenase matrix metalloproteinase1.Together these observations suggest that extract from $M$. citrifolia might be a strong inducer of the biosynthetic activity of extracellular matrix components [3].Therefore, extracts from $M$. citrifolia might contribute to the treatment of not only tumors but also immunological and skin disorders. Psoriasis is a chronic, relapsing, cutaneous condition with $1-2 \%$ prevalence in the general population.Psoriatic arthritis is a chronic inflammatory arthropathy present in $\sim 30 \%$ of patients with psoriasis. In this report, I describe the treatment of psoriasis with $M$. citrifolia, in addition to conventional anti-rheumatism therapy. This is the first report showing the effect of $M$. citrifolia on the skin lesions of psoriasis.

*Address correspondence to this author at the Minami-otsuka Institute of Technology, Minami-otsuka clinic 2-41-9 minami-otsuka, Toshima-ku, Tokyo, Japan; Tel: +81-(3)-3943-7277; Fax: +81-(3)-3943-9018;

E-mail: hokamoto@parkcity.ne.jp

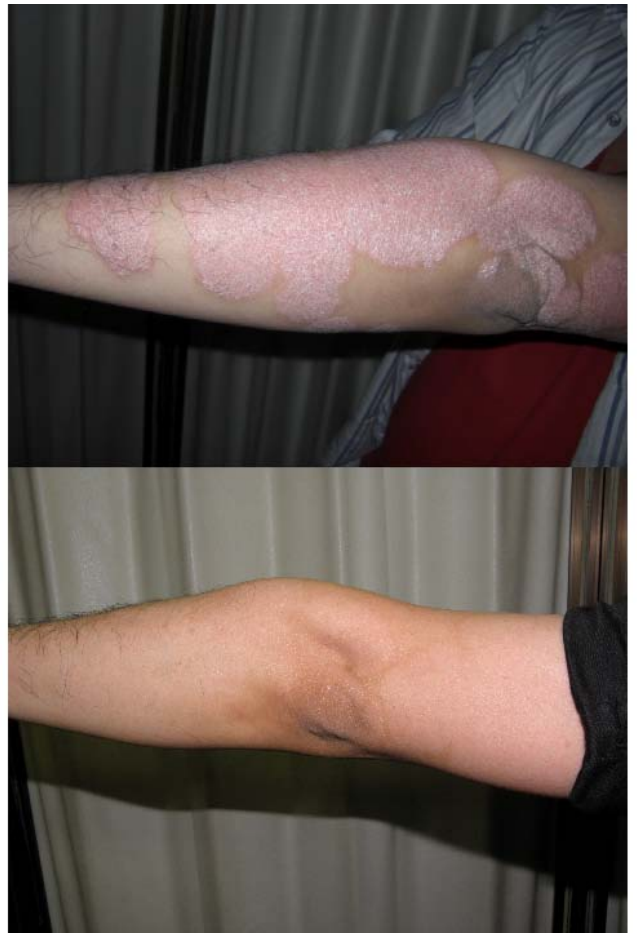

Fig. (1). Skin lesions before the treatment with Morinda citrifolia (Noni) (Fig. 1, upper panel) and after the treatment with $4 \mathrm{~g} /$ day of Morinda citrifolia (Noni) powder together with weekly MTX and no etanercept injections (Fig. 1, lower panel).

A 31-year-old man with a 14-year history of psoriasis developed polyarthritis in 2008 and was admitted to our clinic.Weekly monotherapy with $8 \mathrm{mg}$ of methotrexate(MTX) did not ameliorate his arthritis and treatment with etanercept (25mg every other week) was started in addition to the MTX. Although his economical situation prevented him from using a full dose of etanercept, his arthritis significantly ameliorated and his DAS28-CRP decreased to 1.52. Despite the remission of arthritis, his psoriatic skin lesions were not improved (Fig. 1, upper panel). He then took 4g/day of Morinda citrifolia (Noni) powder together with weekly MTX and ceased taking etanercept 
injections. Although his arthritis worsened after one month, (DAS28-CRP was 2.79), his psoriatic skin lesions surprisingly improved significantly (Figure 1, lower panel). Although the relationship between the $M$. citrifolia and clinical improvement could be non-causal, and this effect might be caused by the effects of concomitant treatment with MTX and etanercept. This is the first report showing the possibility of a clinical effect of $M$. citrifolia on psoriasis.M. citrifolia has been reported to have anticancer and immunemodulation activity $[4,5]$. Therefore Noni, together with MTX might have unknown immune-modulation effects on skin lesions and arthropathy associated with psoriasis.This case also gives us a clue to differentiate between skin lesions and arthropathy in terms of their pathogenesis. This might be explained merely by the strength of immunosuppressive effects, but qualitative differences could also be involved.Further experimental and clinical studies are required to draw a clearer picture of how $M$. citrifolia can modify the pathology of skin lesions associated with psoriasis.

\section{CONFLICT OF INTEREST}

None declared.

\section{ACKNOWLEDGEMENT}

None declared.

\section{REFERENCES}

[1] Hirazumi A, Furusawa E. An immunomodulatory polysacchariderich substance from the fruit juice of Morinda citrifolia (noni) with antitumour activity.Phytother Res $1999 ; 13: 380-7$.

[2] Nayak S, Mengi S. Immunostimulant activity of noni (Morinda citrifolia) on T and B lymphocytes. Pharm Biol 2010 ; 48: 724-31.

[3] Kim SW, Jo BK, Jeong JH, Choi SU, Hwang YI. Induction of extracellular matrix synthesis in normal human fibroblasts by anthraquinone isolated from Morinda citrifolia (Noni) fruit. J Med Food 2005 ; 8: 552-5.

[4] Wang MY, Su C. Cancer preventive effect of Morinda citrifolia (Noni). Ann N Y Acad Sci 2001; 952:161-8

[5] Hirazumi A, Furusawa E. An immunomodulatory polysacchariderich substance from the fruit juice of Morinda citrifolia (noni) with antitumour activity. Phytother Res 1999 ;13: 380-7.

(C) Hiroshi Okamoto; Licensee Bentham Open.

This is an open access article licensed under the terms of the Creative Commons Attribution Non-Commercial License (http://creativecommons.org/licenses/by-nc/3.0/) which permits unrestricted, non-commercial use, distribution and reproduction in any medium, provided the work is properly cited. 